\title{
LA MARGINACIÓN \\ EN LA SOCIEDAD MEDIEVAL. \\ ALGUNOS PROBLEMAS \\ DE MÉTODO
}

\author{
J.E. Ruiz Doménec
}

En los últimos años, historiadores de diversos países se han dedicado a observar la marginación en la sociedad medieval europea. No es mera curiosidad por el mundo del Lumpen. Al contrario, se pretende saber los sistemas de representación social que existen tras este fenómeno. Este deseo es el que ha llevado a crear tipologías de los grupos de marginales y a profundizar en los sistemas de valores propios de ellos. Jacques Le Goff resume el estado de la cuestión y plantea las vías a seguir en estos asuntos ${ }^{1}$. Sus observaciones, siempre pertinentes, orientan bien sobre el tema. Pero leyendo el reciente trabajo de Frantisek Graus, «Randgruppen der städtischen Gesellschaft im Spätmittelalter» ${ }^{2}$ he aprendido lo que nunca había

I J. LE Goff, Les marginaux dans l'Occident médiéval, UGE, Paris 1979, pp. 18-28. (También en II meraviglioso e il quotidjano nell' Occidente Medievale, Laterza, Bari 1983, pp. 165-172. Veánse trabajos de B. GEREMEK, Les marginaux parisiens aux XIV et XV siècles, Paris 1976. Y los reunidos por Guy H. ALLARD, Aspects de la Marginalité au Moyen Âge, Montréal 1975.

${ }^{2}$ En Zeitschrift für Historische Forschung, 1981, pp. 385-437. 
sospechado, ni lo habría hecho aunque hubiera seguido pensando toda mi vida en este problema. Lo único que había hecho era observar las cosas, anotando detalles y hechos fragmentarios, haciendo incluso sencillas generalizaciones y creyendo que no había conexiones entre los hechos diversos, y que el mundo de la marginación era un caos difícil de analizar. Y ahora Graus organiza los conocimientos, elabora sistemáti-camente la realidad de los marginales europeos y nos presenta un universo tan bien delimitado que es conveniente -incluso hasta necesario-seguirle por ese camino.

Graus nos enseña a delimitar con precisión el tema de la marginación en el mundo urbano, a fortalecer nuestra capacidad perceptiva y, en particular, a revalorizar el enfoque topológico, que sugiere la tesis que tras la topografía de la marginación debe buscarse una topología imaginaria. A eso dedicaré las páginas que siguen como un primer intento de abordar este problema y de familiarizarnos con las posibilidades que permite este nuevo enfoque.

Situémonos para ello a finales del siglo XIII y comienzos del siglo XIV; en ese momento se comienza a constituir lo que, en este trabajo, intentaré analizar bajo el apelativo de una topología del miedo. La sociedad bajomedieval (en el sentido técnico de este término, referido, pues, a los siglos XIV y XV) puede ser caracterizada - por razones que explicaré más adelante- como una sociedad cuyo proceso de espacialización se logra a partir del desarrollo de un modelo de conducta, ligado a la naturaleza del miedo ${ }^{3}$. Corresponde a los historiadores establecer con la máxima exactitud este comportamiento social y este reajustamiento en la mentalidad colectiva. Mi propósito es recomponer la historia de un aspecto de esta topología del miedo: el fragmento que relaciona la topografía de las minorías y los grupos marginales (Graus diría Randgruppen) con los aspectos más conmensurables de este fenómeno colectivo. Así pues, restringiré el uso distributivo de este análisis a un aspecto parcial del tema. Mostraré cuáles son las prácticas de exclusión que caracterizan la sociedad bajo-medieval, cuáles son las relaciones de poder que subyacen a estas prácticas de exclusión y cuáles son las

3 J. Delumeau, La peur en Occident, Paris 1978. 
formas de saber, los tipos de conocimiento que emergen en el espacio de esta conducta del miedo, que es la determinante y dominadora de la sociedad de los siglos XIV y XV. En una palabra, la topología del miedo crea inexcusablemente una topografía de la exclusión (creando abierta y decididamente el mundo de los marginales, de las minorías religiosas, de la xenofobia, de la degradación de las culturas periféricas, etc). Éste es, limitadamente, el objeto de análisis del presente estudio.

La formación de la topología del miedo puede ser caracterizada por la aparición, a finales del siglo XIII y principios del siglo XIV, de un hecho que tiene dos aspectos, que son aparentemente contradictorios: por un lado, el cierre exterior de las fronteras de Europa, que coincide con el fracaso de las Cruzadas, la Reconquista y las empresas marítimas en el norte y en el sur; y, por otro lado, la formación de un espacio nuevo, moderno, que, a su vez, se escinde en dos grandes núcleos, el orden flamígero en el norte y oeste de Europa y el llamado Renacimiento en Italia y el Mediterráneo sudoriental. Como se ve, esta aguda transfor-mación no presenta las mismas formas, amplitud y cronología en los diferentes países.

En Inglaterra, por ejemplo, las formas de exclusión comienzan relativamente pronto, pues en 1290 son expulsados los judíos, mientras que las leyes de organización de las comunidades humanas al margen son el resultado de un profundo malestar social, que alcanza a la misma expresión literaria de un Chaucer, quien, en 1386, redacta su Cuento de la abadesa. En Francia, por el contrario, los judíos no son expulsados hasta 1384, y, aunque se produjeron profundas modificaciones en las instituciones civiles frente al fenómeno de los grupos marginales, siguieron por un tiempo conservando cierto poder económico y prestigio social, al igual que en Aragón y en Castilla.

Henos aquí, pues, con un abanico de posibilidades sociales a principios del siglo XIV. La organización del sistema urbano exige necesariamente un proceso de exclusión determinado ${ }^{4}$. La margina-

4 K. Bost, Die Grundlagen der modernen Gesellschaft im Mittelalter, II. Stuttgart 1972, pp. 290 ss. 
ción y la limitación espacial de las minorías es el resultado inmediato (y primero) de este fenómeno, pero viene dificultado a nuestra compresión por cuatro elementos:

1) La cuestión del concepto que la sociedad utiliza para designar a estos grupos de marginales (y propiamente a las minorías). Por sí sólo, el análisis lexicográfico y semántico imposibilita un preciso estudio de estos grupos particulares: los conceptos que aparecen en las fuentes para determinarlos suelen ser más indeterminados que las descripciones de otros grupos.

2) El nivel de la representación. La literatura e incluso la iconografía deben ser aún objeto de precisiones técnicas para fijar la imagen social de estos grupos.

3) Los documentos utilizados son siempre decretos represivos, prohibiciones, reglas, etc., que informan distorsionadamente de la realidad que tratan de presentar.

4) Salvo los judíos, muy pocos de estos grupos crearon una auténtica cultura (en sentido estricto de contracultura, y no - como se dice a menudo- subcultura, pues no es inferior a la «oficial»), por carecer de producción literaria.

Pero, naturalmente, estas graves dificultades no deben impedir que planteemos aquí en toda su amplitud el problema y Jo circunscribamos al tema central de este trabajo: la relación existente entre el espacio y las prácticas de exclusión. Comencemos por orden.

I. En qué consistieron estas transformaciones de las prácticas de exclusión hacia 1300 ? Por una parte, en una reelaboración cuidadosa y detallada de la teoría de la marginación, que alcanzó plenamente el desarrollo penal y la legislación urbana. El principio fundamental de las prácticas de exclusión (que abarca por igual a minorías religiosas y a grupos de marginales) es que existen «personas o grupos (un círculo de personas estructuradas con relaciones precisas entre sí) que no reconocen las normas de la sociedad en la que viven, o bien no las pueden mantener $y$, en base a esta renuncia o incapacidad, no son aceptados como iguales por la mayoría (concepto que por lo demás abarca grupos sociales distintos)». Más técnicamente, la organización del nuevo espacio social (urbano y moderno) exigió la delimitación específica de los grupos que no 
pertenecían a él, creando su auténtica topografía de lo diferente.

El primer principio de exclusión se halla en la imagen creada por el nuevo espacio urbano a finales del siglo XIII. Esta imagen reorganiza los comportamientos individuales y colectivos. En este contex to - lo que Yves Barel llama sistema - será, durante los siglos XIV y XV, el principio organizador de las ciudades y también creador de una verdadera teoría espacial de la exclusión. Ambos elementos forjan una topografía de la diferencia social para los marginales y las minorías religiosas. En otros términos, el sistema urbano bajomedieval se distingue de la ciudad de los siglos XI al XIII por la manera de concebir el espacio y la dinámica social que crea a partir de él, y no estrictamente por las maneras de producción o por los tipos de derechos jurídicos.

Durante estos años se recurre una y otra vez a una representación espacial común, según un uso generalizado, y construye una topografía social mediante la aparición de barrios, concebidos como organismos vivos e integrados y constructivos en los que se reúnen natural y espontáneamente diferentes grupos sociales por afinidades. Así, los artesanos se van concentrando y dan nombre a las calles. Este proceso de espacialización conduce a dos consecuencias opuestas: de una parte, existe el espacio de los grupos «integrados» (no privilegiados, pues el concepto de privilegio es muy amplio en esta época), donde el poder de la ciudad (y más allá de él, el poder monárquico) se ejerce de un modo activo y positivo, favoreciendo la implantación y el desarrollo de la máquina integradora (aceptan inmigrantes en grupos homogéneos, y propiamente extranjeros; existen en numerosas ciudades barrios de italianos, etc.). Y, de otro lado, la afirmación del proceso mismo de la exclusión. Se trata de definir con precisión el lugar ocupado por los marginales (sean quienes sean) y con ellos, el de las minorías (fueran cuales fueran): aislarlos mediante medidas que tienden públicamente a identificar estos grupos con la delincuencia generalizada en esta época. Por lo demás, no imaginemos que esa representación espacial sea fácil de detectar en las fuentes de la época. La dificultad de encontrar documentos que den a la vez una idea de las fortunas de los individuos y su localización es un fenómeno extremadamente difícil, incluso para períodos mucho más modernos, donde los criterios sociológi- 
cos se unen a los meramente urbanísticos. Pero se tiende a encontrar un espacio determinado para estos grupos "no integrados» (ampliamente el margen, y que se desarraigan abiertamente de las líneas marcadas por el poder). Esta delimitación de un espacio del poder y de uno que atenta al poder comienza a caracterizar la historia de Occidente. Veamos algunos rasgos de este fenómeno, centrado exclusivamente en el problema de la topografía urbana:

1) La decisión política. Debería estudiarse con detención cuándo se dispuso oficialmente el ghetto para la población judía, y si existe una clara intención de aplicar este criterio de "ghettoización» para el resto de los grupos marginales y de las minorías religiosas. Este espacio establece un tipo de relación con el conjunto social desde un punto de vista negativo: rechazo, exclusión, desestimación, barrera, y aun ocultación o degradación. El poder de las ciudades (y el monárquico) trató de delimitar a los individuos en espacios cerrados, propios de ellos, y con tendencia hacia su sistematización. Los efectos de este proceso adquieren la forma general del límite social y de la carencia de privilegios públicos, de incardinación en el aparato del naciente Estado.

2) La justificación del espacio de exclusión como una barrera necesaria. El poder de la ciudad, esencialmente, trató de inculcar en la población integrada la idea de que la conducción de los grupos marginales a esos espacios particulares era una necesidad política. Los marginales, incluso las minorías, que en esto no se diferenciaban del resto, se dejaron arrastrar a estos espacios especiales por su continua tendencia a la criminalización de sus actos. Por ejemplo, a mediados del siglo XIV se acusó a los judíos de actos criminales, justificadores de su exclusión espacial, como el haber envenenado las fuentes en el período de las grandes pandemias, etc.

3) La extensión del ciclo espacial. Estas medidas de establecer una topografía espacial para los judíos (que naturalmente terminaron con su destierro definitivo y su expulsión de las ciudades y más tarde de los mismos países) se incrementan para el resto de grupos marginales y minorías. El poder trató de establecer en todos los casos una ley drástica de la prohibición de una movilidad social dentro de las ciudades. Los barrios pobres, y marginales, de prostitutas, 
de leprosos o de los simples lugares del crimen comenzaron a aparecer en las ciudades a mediados del siglo XIV.

El segundo principio de exclusión, referido casi exclusivamente a los judíos (y más tarde a los gitanos) es que las leyes positivas formuladas por el poder cívico, urbano y político, monárquico, para ser consideradas buenas, no deben ser cuestionadas por nadie. Transcriben espontáneamente una relación entre la ley y el orden natural, moral y religioso. La ley debe ser aceptada por todos por igual; en este sentido, son leyes de uniformización de la sociedad (en la que los judíos se veían como grupos diferentes, grupos-otro - Graus los denomina Anderssein), desde su origen, y que de algún modo quieren ser conscientemente distintos a la sociedad en la que viven, ya sea en sus creencias (aspecto religioso de la ley), ya sea en sus normas de vida (aspecto moral de la ley) o en su origen biológico (aspecto natural de la ley). Esta clara distinción de los judíos frente a los otros grupos marginales (prostitutas, pobres, lisiados, criminales, etc.) hace posible la formación de verdaderas contraculturas. En este sentido, toda investigación topográfica debería tener presente los siguientes elementos:

1) Las relaciones entre cristianos y judíos antes de los «progroms». Es decir, entre 1270 y 1330 , aproximadamente.

2) El proceso de degradación del judío conforme iba avanzando un tipo peculiar de religiosidad y de piedad popular que introducía el demonio como la base de toda situación de otredad. Los judíos fueron «demonizados» porque no podía haber nada «natural» fuera del nuevo orden social.

3) Fijar la naturaleza exacta, para estos años, de la frase de Sartre de que «los judíos son una creación de los cristianos», al provocar en estos años una textura espacial que imposibilitaba su asimilación.

El tercer principio de exclusión se deduce claramente de los dos primeros: una definción clara y simple del concepto «minoría» religiosa se liga al conocimiento de las prácticas de exclusión y de marginación. La topografía es un fenómeno de organización de un sistema ideológico previo y de una presión popular cada vez más 
delirante y eficaz. Naturalmente, esta actitud constituye un daño social irreparable, una perturbación en el proceso de civilización de la sociedad europea, una incomodidad para el conjunto de la sociedad; es decir, escapa a la observación primaria del historiador, ligándose a fenómenos poco conmensurables. Presentes, pero constantemente diluidos en las fuentes. Esta atmósfera mental (impulsada por el miedo) es la que, con mayor vigor, exige la delimitación espacial de las minorías religiosas y de los grupos marginales. De todos modos, tal como Jean Delumeau reconoce, la marginación y la exclusión de las minorías religiosas es algo emparentado a partir del siglo XIV con el pecado y la nueva dimensión de la piedad popular, con el sentimiento de culpa y con la idea de falta. Se puede pertenecer a este espacio concreto del sistema urbano por tres razones, que por sí mismas delimitan las prácticas de exclusión y de marginación, y definen el valor de "minoría» religiosa, que son:

1) Haber nacido en el seno de un grupo diferente (como los judíos y ulteriormente los gitanos).

2) Entrar pasivamente en él (los enfermos, los pobres, los mendigos).

3) Entrar activamente en él (criminales, prostitutas, etc.).

Como se ve, lo único que les define es el espacio, con diferentes tiempos de exclusión. La definitiva, como la de los judíos; la provisional, como la de los pobres; la radical, como la de los criminales. Por eso conviene estudiar, como afirma Graus, "la cuestión de qué incumplimiento de normas tenía efecto de marginación».

Veo dos casos, plenamente diferenciados: uno es la infracción a la ley natural, a la ley religiosa, a la ley moral; otro, diferente, donde la infracción es la ruptura voluntaria con la ley civil, explícitamente desarrollada por el poder urbano y monárquico hacia 1300: en este segundo grupo hay que introducir a todos los criminales, esos individuos que componen el Lumpen proletariado del mundo urbano de los siglos XIV y XV. Es evidente que las sutiles fronteras entre un tipo y otro fueron muy a menudo suprimidas, y los efectos de una situación espacial al margen fueron considerados el resultado simple de un «crimen» social (por ejemplo, los «progorms» de 
finales del siglo XIV fueron en gran parte resultado de esta voluntaria confusión) o de un «crimen» económico (Braudel ha establecido una extraña correlación entre la coyuntura económica y demográfica y las persecuciones, las masacres, las explusiones y las conversiones forzadas). El ejemplo inicial es el de 1320 en el Midi de Francia, con el movimiento de los pastereaux, donde al parecer se exterminaron 140 comunidades judías por masas campesinas asoladas por el hambre y las epidemias (enloquecidas por una ira sorda y delirante). Estos «pastores» - como señala Rodney Hilton-constituían por lo general el grupo más juvenil y de mayor movilidad entre la población rural»5 ${ }^{5}$.

II. Tal es ciertamente el primer ámbito de estudio. Ahora conviene dirigir la mirada a otra esfera de observación: aquélla que liga esta nueva práctica social y las transformaciones en la esfera imaginaria. He aquí una cuestión mucho más ardua y comprometida. Se puede plantear del siguiente modo: ¿cuáles son, de dónde vienen y a qué responden estos mecanismos de exclusión? Desde finales del siglo XIII, comenzando - repito- por Inglaterra, y más tarde Francia, Castilla, Aragón y Alemania, etc., se forman, en niveles relativamente bajos de la escala social, grupos de individuos que cuestionan constantemente la tolerancia de las clases elevadas (de la «buena» sociedad, e incluso del propio rey) ente la diversidad social, cultural y religiosa de su momento. El escándalo de banqueros judíos como financieros de los reyes consagra este movimiento «popular». Lo exalta. Crea el mecanismo para la organización de una protesta colectiva. En las ciudades el pueblo grita contra esta connivencia. Los predicadores lo apoyan. La jerarquía de la Iglesia presiona al poder público. La respuesta inmediata por parte de ese poder fue «satisfacer las demandas populares» (en el sentido medieval del término), fabricando un enemigo cómodo y fácil de situar en un lugar del sistema social. La topografía de las minorías y la marginación fue el resultado inevitable de una transformación revolu-

5 R. HiLton, Bond Men Made Free. Medieval Peasant Movements and the English Rising of 1381. Viking Press, 1973. 
cionaria en los sistemas de valores y de las conductas de los individuos, y del contacto que las capas populares tuvieron frente al mundo exterior y al «interior-extraño». Hacia 1300 se comprueba en toda Europa, especialmente en los centros urbanos, lo siguiente:

1) Que las «válvulas» sociales no funcionan.

2) Que existen escasas posibilidades de ascenso social y mucho mayores de descenso. La Baja Edad Media polarizó la sociedad hasta el extremo de convertir a los ricos en más ricos y a los pobres en más pobres.

3) Que se agudizan los problemas sociales, con el hundimiento de las fronteras de la tolerancia.

4) Que se plantean reacciones alérgicas ante lo diferente, y se adoptan formas histéricas ante el mundo del trabajo intelectual (como en el caso de los judíos).

Éstos son, estrictamente hablando, los mecanismos que hicieron posible este crecimiento insospechado de los sistemas de exclusión social y la aparición de una conducta negativa ante los marginales y las minorías.

De dónde vienen. Este tema es más complejo y controvertido. Los historiadores debaten entre sí. No existe unanimidad de criterios. Según un orden cronológico, lo primero son indicios de una "sensibilidad popular» extremada y exacerbada frente a los enemigos, los diferente: el miedo, cuyo tópos se estaba forjando en estos mismos años ayudó profundamente a este sentimiento. La nueva tendencia religiosa, y el cristianismo «popular» ayudó a forjar el espíritu de la marginación y a excluir a las minorías. El cinismo fue la coartada moral de los predicadores.

A qué obedecen. Éste es realmente el nudo de la cuestión. Deberá analizarse centrándolo en el problema de los judíos, que es, ha sido y será, el tema par excellence de todos cuantos pueden enfrentarse en esta proyección de la ideología de cara a la delimitación de un espacio de exclusión.

Quisiera, para terminar (y una vez completado el análisis y configurado las líneas que podrían llevar a cabo una topografía social de las minorías religiosas y de la marginalidad) extraer algunas concluisones provisionales que intentaré utilizar como indicios para 
una nueva profundización topológica del análisis topográfico de las minorías.

Los datos controvertidos (y que deberán ser objeto de debate entre los especialistas en el tema) son los siguientes: $\measuredangle$ Cómo fue posible que el conjunto teórico de las reflexiones sobre la exclusión quedara de hecho desordenado y encubierto por una práctica religiosa confusa, contradictoria, totalmente especulativa y que logró reelaborarse incluso por los grandes humanistas y reformadores de los siglos XIV y XV cuando se siguió insistiendo en el presente tema? ¿Cómo pudo dar la sociedad europea ese paso de gigante ante los principios de exclusión, olvidando la gran lección de sociabilidad de los siglos XII y XIII, relegando la actividad creativa y finalmente dejar al mundo ante el mismo miedo, mediante una práctica de coerción penal (la Inquisición) basada en un análisis parcial y perverso de los comportamientos individuales y dirigida fundamentalmente a corregir cualquier actividad de diferenciación? ¿Cuál es el origen y la meta que persiguen los grupos dominantes y dirigentes en la sociedad europea con tales prácticas de exclusión y que determinan la topografía de las minorías y los marginales y el nuevo sentido constructivo impuesto por el estyle flamboyant? Al parecer, la Baja Edad Media, para escapar al miedo, se creó para sí misma unos instrumentos de autorrepresentación suficientemente sólidos y refinados que permitieron soportar el asalto del enemigo y no destruir la sociedad. Quizás sea cierto que «cuanto más articulada está realmente una obra, tanto más se dice de su misma concepción; viniendo en ayuda de la mímesis lo que es su contrario». La histeria y la neurosis son el rasgo determinante de una espiritualidad que no permite la diferencia y que prefería a buen seguro la articulación delirante del mundo al tratamiento dialéctico de los problemas. Pero, paradójicamente, en esta profunda inflexión del comportamiento humano, que creó una verdadera topografía de las minorías y la exclusión, debe verse de seguro el origen del capitalismo.

Pero éste es otro asunto. Mi propósito ha consistido en describir con brevedad una especie de consideración sobre el espacio de exclusión en el que se introdujo a un número indiscriminado de «minorías", ilustrado con algunos ejemplos y con algunos planteamien- 
tos para futuras investigaciones. Mi argumento, desplegado de una forma angular y difícil (el tema en sí mismo lo es), vuelve al punto de partida. La sociedad urbana de la Baja Edad Media necesitó de la marginación para articular coherentemente un sistema de representaciones colectivas asentado en el privilegio de pertenecer a una función, una etnia o un grupo. De este modo, se libera del miedo, y, como dice Jacques Le Goff en un libro ya clásico, aleja de sí todos los males que veía en estos Randgruppen. Después viene la pena. Y lo demás. 